\title{
Human-machine alliance against cervical cancer
}

\author{
Igor Govorov (D) , St Petersburg, Russian Federation, Stockholm, Sweden; Elena Dikareva, St Petersburg, Russian \\ Federation; Elena Velichko, St Petersburg, Russian Federation; Vesna Kesic, Belgrade, Serbia and \\ Eduard Komlichenko, St Petersburg, Russian Federation
}

In Russia, cervical cancer presents a major problem in women's health, since the incidence and mortality rates have not decreased compared with other European countries. The reasons are insufficient coverage by vaccination against human papillomavirus and flaws in screening. As a result, there were almost 17000 cases of cervical cancer in 2018, with an estimated age-standardized incidence rate of $17 / 100$ 000 women. In addition, geography also contributes to be the problem, because Russia, being the largest country globally, has many regional concentrations located at great distances from each other. All this determines the need to use non-standard approaches that are different from those used in countries with close urban locations. As a possible solution to the problem, one may use the latest computer technologies not impacted by distances and provide process information at a very high speed.

The Almazov National Medical Research Center is the major healthcare provider and scientific center, located in St Petersburg, Russia (Figure 1). With more than 1,500bed capacity, wet-lab, and animal facilities, Almazov strives to accelerate the path from the bench to bedside for the most promising technologies. Here we have developed a machine learning system, able to detect cervical lesions. Our project developed in collaboration with Peter the Great St Petersburg Polytechnic University - the leading Russian educational and scientific facility. The program started in 2019 and currently involves four clinics located in St Petersburg. At the same time, we will be glad to cooperate with other clinics both from Russia and globally.

The main goal of our software is to help the clinician in decision-making. The workflow includes colposcopic image processing, targeting the suspicious lesions, and analyzing them by a trained artificial neural network. The first step is

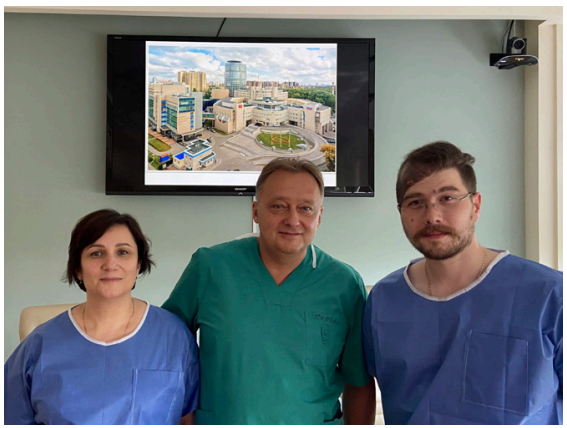

Figure 1 Almazov National Medical Research Center team: from left to right, E.Dikareva, E.Komlichenko, I.Govorov.

to collect the colposcopic images, and since the performance improves together with new data input, we aim to retrieve as many images as possible. Then, we divide the images into a training set and test set, the first is uploaded into the convolutional neural network VGG-16. The images go through three subsequent stages: detection, segmentation, and classification. Detection aims to define the principal area and highlight it with a bounding box (Figure 2). Segmentation is cutting the image into several meaningful segments, which usually correspond to different tissues or lesions. Classification puts an image into one of the prespecified classes.

In the preliminary phase, we evaluated performance in classifying the images into either 'Healthy' or 'Unhealthy' groups. The accuracy equalled $96 \%$. Now, our work continues in two main ways. On the one hand, we test different artificial neural networks and compare performance while simultaneously improving the detection accuracy through subdividing images into low/high grade-squamous intraepithelial lesions or miscellaneous findings.

This work would not have been possible without the contribution from all our colleagues. We want to express our special gratitude to Professor Vesna Kesic for her

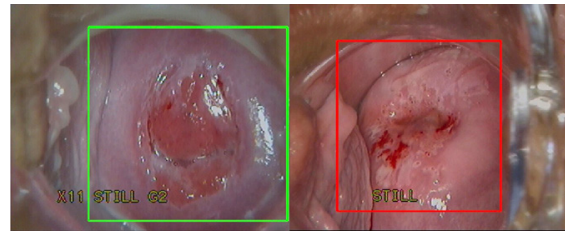

Figure 2 An example of image processing. The program automatically squares the areas of interest, with the green color indicating the normal cervix, and the red color the presence of a lesion, in this case high grade.

support and participation in the development of this project. The first results were promising, and we cannot wait to continue working on the project. We are confident that this approach will find its application in many other centers.

Correspondence to Dr Igor Govorov, Institute of Perinatology and Pediatrics, Almazov National Medical Research Center, St Petersburg 197341, Russian Federation; govorov.igor.med@gmail.com

Contributors IG, ED, VK, EK: collecting and classifying the colposcopic images. EV: image processing with ANN. All authors have contributed to writing the manuscript and reviewing it.

Funding The authors have not declared a specific grant for this research from any funding agency in the public, commercial, or not-for-profit sectors.

Competing interests None declared.

Patient consent for publication Not required.

Provenance and peer review Commissioned; internally peer reviewed.

(c) IGCS and ESGO 2021. No commercial re-use. See rights and permissions. Published by BMJ.

D Check for updates

To cite Govorov I, Dikareva E, Velichko E, et al. Int J Gynecol Cancer 2021;31:299.

Accepted 15 September 2020

Published Online First 30 September 2020

Int J Gynecol Cancer 2021;31:299.

doi:10.1136/ijgc-2020-002064

ORCID ID

Igor Govorov http://orcid.org/0000-0003-1809-0270 